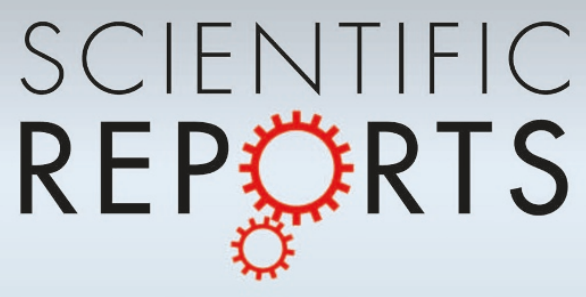

OPEN

SUBJECT AREAS:

ELECTRONIC DEVICES

ELECTRICAL AND ELECTRONIC

ENGINEERING

Received

13 May 2014

Accepted

26 September 2014

Published

5 November 2014

Correspondence and requests for materials should be addressed to J.K. (joonkim@ incheon.ac.kr)

\section{Incident light adjustable solar cell by periodic nanolens architecture}

\author{
Ju-Hyung Yun', Eunsongyi Lee ${ }^{2}$, Hyeong-Ho Park ${ }^{3}$, Dong-Wook Kim², Wayne A. Anderson', \\ Joondong Kim ${ }^{4}$, Natalia M. Litchinitser' ${ }^{1}$ Jinwei Zeng' ${ }^{1}$ Junsin $\mathrm{Yi}^{5}$, M. Melvin David Kumar ${ }^{4}$ \& Jingbo Sun'
}

'Department of Electrical Engineering, University at Buffalo, State University of New York, Buffalo, New York 14260, USA, ${ }^{2}$ Department of Physics, Ewha Womans University, Seoul 120750, Korea, ${ }^{3}$ Patterning Process Department, Nano Process Division, Korea Advanced Nano Fab Center, Suwon 443270, Korea, ${ }^{4}$ Department of Electrical Engineering, Incheon National University, Incheon 406772, Korea, ${ }^{5}$ College of Information and Communication Engineering, Sungkyunkwan University, Suwon 440746, Korea.

Could nanostructures act as lenses to focus incident light for efficient utilization of photovoltaics? Is it possible, in order to avoid serious recombination loss, to realize periodic nanostructures in solar cells without direct etching in a light absorbing semiconductor? Here we propose and demonstrate a promising architecture to shape nanolenses on a planar semiconductor. Optically transparent and electrically conductive nanolenses simultaneously provide the optical benefit of modulating the incident light and the electrical advantage of supporting carrier transportation. A transparent indium-tin-oxide (ITO) nanolens was designed to focus the incident light-spectrum in focal lengths overlapping to a strong electric field region for high carrier collection efficiency. The ITO nanolens effectively broadens near-zero reflection and provides high tolerance to the incident light angles. We present a record high light-conversion efficiency of $16.0 \%$ for a periodic nanostructured Si solar cell.

M any approaches have been reported to achieve low-cost ${ }^{1-7}$ and high-performing photovoltaics (PVs) using designs for light-incident surficial structures ${ }^{3,8-13}$, and/or optical behaviors ${ }^{14-18}$. Recently, great interest has been focused on the periodic structure of light-active materials for optical enhancement ${ }^{19-22}$. Lambertian light trapping is proposed for an ideally random surface, which can extend the optical path length by $4 \mathrm{n}^{2}$ or higher ${ }^{21,23,24}$, where $\mathrm{n}$ is the refractive index. A strong candidate for extraordinary optical enhancement by $14.5 \mathrm{n}^{2}$ has been proposed ${ }^{25,26}$ using a periodic nanoscale grating structure ${ }^{27}$.

Microscale designs are attractive for fabricating structures using a commercial method for large scale application $^{11-13}$. Nanoscale architectures are definitely promising in attempts to reduce light-reflection with an enormous enlargement of the light-active surface area; however, it is extremely difficult to establish a neutral region ${ }^{28,29}$ or a space charge region (SCR) ${ }^{11,13,30}$ for nanoscale entities due to their tiny geometry. The SCR has the highest carrier collection efficiency, benefitting from a strong electric field $(E)$ existing inside the region. Recent investigations have revealed that the position of an SCR is peculiarly crucial inside light-absorbing entities ${ }^{11,13}$.

Though the ITO nanowire and nanorod structures have been employed in photovoltaic and LED applications ${ }^{31-33}$, there are limitations in shaping the junctions of the nanoscale devices ${ }^{9,34}$. A direct etching method has been typically used to pattern semiconducting material. However, the direct etching of a semiconductor almost always leads to surface defects $s^{3,24,27,28,30,35}$. Such defects in the semiconducting material readily cause low carrier collection efficiency ${ }^{34}$, resulting in degraded solar cell performance of $\sim 10 \%$ or less for Si and $13.8 \%$ for compound semiconductors; those achievements $s^{5,27,28,30,36}$ are far behind from the pursued efficiency of over $20 \%$ of Si solar cells $s^{6,22,35,37}$.

The defect-induced recombination loss is a main reason to cause a discrepancy between the optical benefit and the electrical degradation of the direct nanoscale patterned semiconductor PVs. And thus, an urgent issue is assigned to the nanostructured PVs active light management ${ }^{23,38}$. Is it possible to form the optically beneficial nanostructures without electrical recombination loss by surface defects of the direct patterned semiconductor?

One promising scheme can be a periodically nanoscale patterned transparent conductor. In this design approach, it is possible to assign nanostructures for a planar semiconductor without a need of the direct etching in semiconducting material.

In this paper, we propose and demonstrate a periodic nanoscale-patterned high performing Si solar cell, without direct etching in a light-active semiconductor. This design is realized by an imprint method to form nanoscale transparent patterns on a bare 4 -inch Si wafer. 
In this paper the following challenges are addressed:

Fabrication of the periodically nanostructured PV device without a direct etching of the semiconducting material.

Optically transparent indium-tin-oxide (ITO) nanolenses were periodically formed on a planar $\mathrm{Si}$, which enables to fabricate the nanostructure PV device without damage to the semiconductor. And thus, the light-absorbing semiconductor ( $\mathrm{Si}$ ) is free from the surface defects, which are readily caused by the direct etching of the semiconductor. This approach ideally provides no etching-induced recombination loss from the semiconductor.

Optical and electrical functions of the ITO nanolens

The incident light has a form of plane wave and passes a planar semiconductor without an optical modulation. We have optimized the ITO nanolens to work as an optical lens by tuning the propagation length of the incident light. The nanolens geometries were designed to focus the various photon wavelengths at a designated spot to maximize the collection efficiency of photogenerated carriers. In terms of an electrical aspect, the electrical conducting ITO nanolens-arrays support the photo-generated carrier transport.

Reflection minimization for the incident photon wavelengths To improve solar cell performances, it is essential to drive the incident light into a light-absorber with little reflection at the surface. The ITO material has an intermediate refractive index value between air and Si. And thus, an ITO layer insertion spontaneously reduces the light reflection. We demonstrated an effective scheme of near-zero reflection for a broad range of wavelengths by using the ITO nanolenses.

High tolerance to the incident light angles

Not only is the solar cell efficiency important, but also it is a crucial issue to extend the solar utilization time, which directly increases the solar power generation. Unfortunately, the incident light angle to a solar cell varies with time during the day and throughout the year. This limits the power generation time for a conventional solar cell. The ITO nanolenses-embedded solar cell shows a strong potential of the high tolerance to broad incident light angles.

A spatial overlapping of the photo-generated carrier region and the SCR location to enhance the carrier collection efficiency.

The incident light generates the photo-generated carriers in the light-absorbing Si material and establishes the lightinduced $E$. For a solar cell, there exists a built-in $E$ in the SCR, which is a driving force to collect photo-generated carriers. It is reported that few of the nanostructure systems such as metallic nanospheres ${ }^{39}$, silver nanorods ${ }^{40}$ have been used to focus and magnify the broadband color images through localized surface plasmon (LSP) resonance. In the present work, a nanolens system is applied in renewable energy generation. Hence, the nanolens was designed to a focus broadband solar spectrum over the SCR rather than transfer the specific color and magnitude information for imaging. The more important geometric factor of the nanolens is the radius, which determines the focal length of the light. The spatial overlapping of the light-induced $E$ and the built-in $E$ due to focusing the photon wavelengths over SCR enhances the carrier collection efficiency ${ }^{41}$.

\section{Results}

Formation of nanolens-arrays. Figure 1 shows the schematic of the proposed structure. Nanostructure features were embedded in an optically transparent ITO layer to fabricate nanolenses, instead of using a Si substrate. This approach properly formed the periodically nanostructured PV device without a direct etching process to Si.
In order to fabricate the proposed ITO nanolens structure, we used a nanoimprint method to fabricate nanoscale patterns, which is suitable for patterning of large-scale periodic structures with low fabrication cost and high throughput ${ }^{42}$. Our nanosize hole-array was patterned on a 4 -inch Si wafer as a target substrate. A polymer mold (polyurethane acrylate, PUA) was used to inversely replicate the structure from a master stamp (Fig. 1a). The UV imprinting process was performed to pattern hole-arrays on the target Si substrate (Fig. 1b). The inversely replicated PUA mold was then pressed against the UV-curable resin. By detaching the PUA mold from the UV-irradiated resin, the hole-arrays of the imprinted resin layer were produced on a $200 \mathrm{~nm}$-thick polymethyl methacrylate (PMMA) layer. Subsequently, an $\mathrm{O}_{2}$-etching process was used to pattern identical hole-arrays on a PMMA layer (Fig. 1c, d, e).

We deposited a $200 \mathrm{~nm}$-thick ITO film on the PMMA hole-array Si substrate. A lift-off process was performed to remove the PMMA layer in an acetone solution under $15 \mathrm{~min}$ of sonication; there remained a $200 \mathrm{~nm}$-height ITO-nanolens (with a radius of $180 \mathrm{~nm}$ ) array on the Si substrate (See Methods), as presented in the top-view image (Fig. 1f) and in the cross-sectional view image (Fig. 1g). Each ITO-nanolens spontaneously contacts to the Si substrate; however, it is electrically isolated from the neighboring nanolenses. An additional an ITO film $(80 \mathrm{~nm})$ was coated on the previously formed $200 \mathrm{~nm}$-height ITO-nanolens-arrays on the $\mathrm{Si}$ substrate (Fig. 1h). A cross sectional image (Fig. 1i) clearly depicts the ITO-film coating layer on the ITO nanolenses and the Si substrate, which ensures the electrical connection through the entire nanolens-arrays.

As a result, the front surface has nanoscale patterns comprised of an $80 \mathrm{~nm}$-thick ITO film layer with $200 \mathrm{~nm}$-height ITO-nanolensarrays. Electrical conduction was obtained from this front surface; the device was found to have a low sheet resistance of $29.4 \Omega / \mathrm{sq}$, which will ensure good electrical contact ${ }^{12,43,44}$. The optical transmittance was measured and found to be $90.95 \%$. Photographic images are presented for the ITO nanolens-array patterned on the 4-inch Si wafer (Fig. 1j) and the glass substrate (Fig. 1k).

Reflection profiles for the flat ITO film, nanolens-arrays, and nanolens-arrays with ITO film-coating on a $\mathrm{Si}$ substrate. In order to characterize the ITO-nanolens structure, we performed the following optical and electrical measurements. A reduction in reflection is crucial to drive more photons into a light-absorber. We measured reflectance profiles from various ITO structures on a $\mathrm{Si}$ substrate (Fig. 2a). A flat $280 \mathrm{~nm}$ ITO film was prepared to compare the reflection features of a $200 \mathrm{~nm}$-height ITO nanolens-array (Nanolens) and a $200 \mathrm{~nm}$-height ITO-nanolens-array with a coating of $80 \mathrm{~nm}$-thick ITO film (Nanolens $+80 \mathrm{~nm}$-ITO film). The thickness of the additional $80 \mathrm{~nm}$ ITO coating film was chosen using an optical interfacial design for bare Si (See Methods), in order to minimize the reflectance ${ }^{28,44}$.

Average reflectance was found in the range of $400-1100 \mathrm{~nm}$. A bare $\mathrm{Si}$ substrate gave a high reflection value of $38.90 \%$. Meanwhile, a $280 \mathrm{~nm}$-ITO film embedded Si substrate moderately reduced the reflection value to $18.63 \%$. A nanolens-embedded surface more effectively reduced the reflection value (13.85\%). A minimum reflection point $(5.39 \%$ ) was found at $\lambda=510 \mathrm{~nm}$, without touching the zero reflection point.

A further reduction of the reflection of $4.70 \%$ was achieved from a nanolens with an ITO film-coating surface. The additional ITO filmcoating fully covered the surface without leaving behind a bare $\mathrm{Si}$ region. It is worthwhile to note that this structure provides broad near-zero reflection $(\mathrm{R}<1 \%)$ for wavelengths between $608 \mathrm{~nm}$ and $751 \mathrm{~nm}$.

A practical solar cell has a peak electric power generation condition, in which the light incident angle is normal to the solar cell, having a zero angle value. However, the incident angle is time-varying 
a

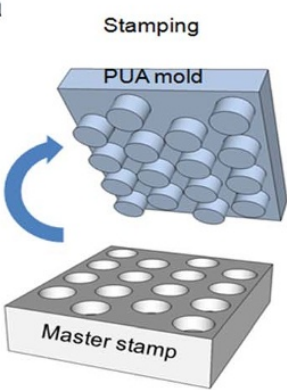

e

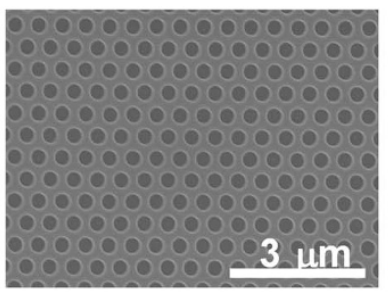

b

UV imprinting

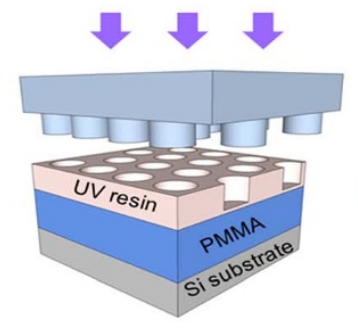

f

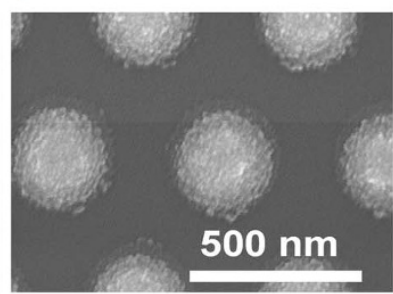

j

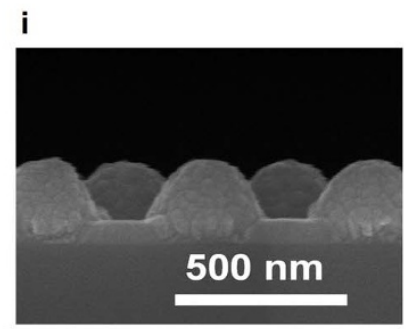

C

$\mathrm{O}_{2}$ etching

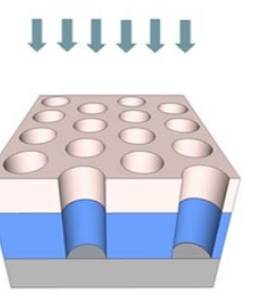

g

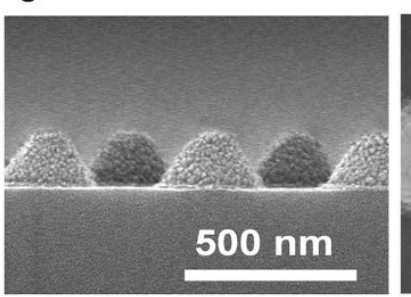

d

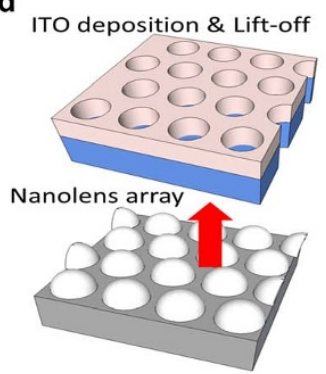

h

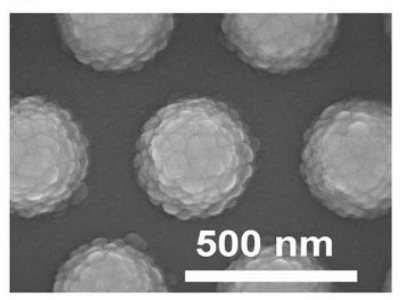

k
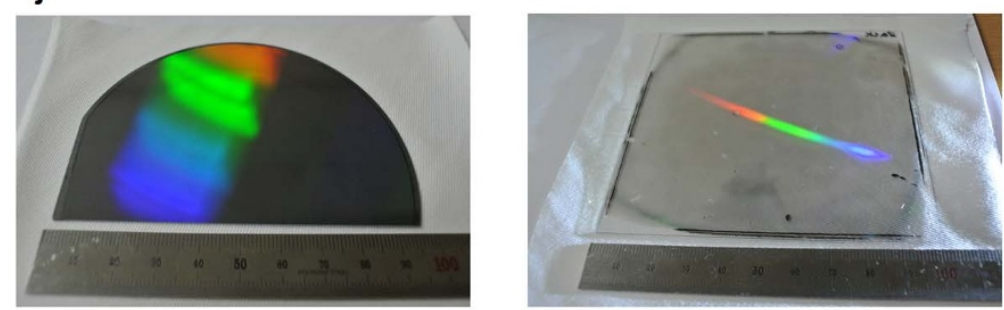

Figure 1 Formation of nanolens-arrays. (a-d), Schematics of nanoimprint method. (e), A scanning electron microscopy (SEM) image of PMMA holearrayed patterns. SEM images of ITO-nanolens-arrays (f, g), and ITO-nanolens-arrays with an additional $80 \mathrm{~nm}$-thick ITO film (h, i). Photos of ITOnanolens-arrays on Si wafer (j) and on glass (k). For the Si wafer, the n-p junction was previously formed using a doping process (See Methods).

and therefore a sustainably high tolerance of the independence to the incident light angle is an important factor ${ }^{8,10,18,21,45}$ to improve the solar cell power-generation amount and the utilization time as well.

An ellipsometric system was used to measure the angle-dependent reflectance profiles for the flat ITO film, nanolens-arrays, and nanolens-arrays with ITO film-coating on a Si substrate with incident angle variations from $30^{\circ}$ to $75^{\circ}$; data are presented as color maps (Fig. 2b-d). The flat ITO film has very limited wavelength range for zero-reflection and showed substantial reflectance profiles for shortwavelengths, indicating crucial angle dependencies. Meanwhile, the nanolens surface seems to be efficient at reducing reflectance values for wavelength variations. In addition, this structure sufficiently relieves the incident angle dependency. A further improvement has clearly been shown with the nanolens-arrays with ITO film. This structure significantly expands the near-zero reflection regions with a high tolerance to incident-light angles for broader wavelengths (Supplementary section 'Weighted- reflectance').

Light distribution according to wavelengths. The transparent nanolens structure has a convex feature, and thus can work as a lens. In conventional optics, focusing size and depth are limited by diffraction phenomenon ${ }^{46,47}$. A solid immersion lens has been shown to have the potential to overcome the diffraction limit by filling an object space with a solid material, that has a high-refractive index ${ }^{47-50}$.

The ITO-nanolens can provide an active solution for efficient light management; it can focus the incident light into a light-absorber. When a plane wave is incident on the ITO-nanolens, the light is refracted by the interface curvature and establishes a length that can be focused. We have investigated the focal length $\left(\mathrm{F}_{\text {lens }}\right)$ formation for short, medium, and long wavelengths, as shown in Table 1 (also see Methods).

The behaviors of refraction tuning are directly related to the $F_{\text {lens }}$ formation, which is determined according to wavelength variations (Supplementary section 'Refraction tuning'). In the visible range, the ITO-nanolens effectively modulates the light propagation, focusing the shorter wavelength light into a deeper Si absorber and the longer wavelength light into a thinner Si absorber (Fig. 3a).

The light-induced electric field $\left(E_{\text {light }}\right)$ intensity distribution in a $\mathrm{Si}$ absorber is controlled by two factors. First, the absorption coefficient of $\operatorname{Si}\left(\alpha_{\mathrm{si}}\right)$ is high at short-wavelengths ${ }^{51}$, resulting in fast decay of the field intensity in Si. Second, the $\mathrm{F}_{\text {lens }}$ of the nanolens is gradually decreased, with increased wavelengths in the visible range (Table 1), due to the reduction of the refractive index of $\mathrm{Si}\left(\mathrm{n}_{\mathrm{Si}}\right)$.

In order to investigate the optical interaction and the $E_{\text {light }}$ distribution of the ITO-nanolens-embedded $\mathrm{Si}$, a numerical simulation based on a finite-difference time-domain (FDTD) calculation (Supplementary section 'FDTD simulation') was performed for the ITO-nanolens/Si unit cell according to various wavelength photons (Fig. 3b-e). Comparison results are also presented for the flat-ITO film on the Si substrate (Fig. 3f-i). For the flat ITO film, the field magnitude is readily exponentially decayed in the Si absorber due to the increasing distance from the surface (Fig. 31). In contrast, the plot of $E_{x} / E_{0}$ of the ITO-nanolens array has characteristic patterns by modulating of the incident light, demonstrating the tendency of a strong $E_{\text {light }}$ to appear along the $\mathrm{z}$-direction of the Si depth (Fig. 3j,k).

The ITO front surface consists of the nanolens-arrays. Thus, the light focused by the nanolens will meet the light from the neighboring nanolenses, which can cause interference. As a result, the lens array will generate second and third peaks (Fig. 3a). The FDTD 
a

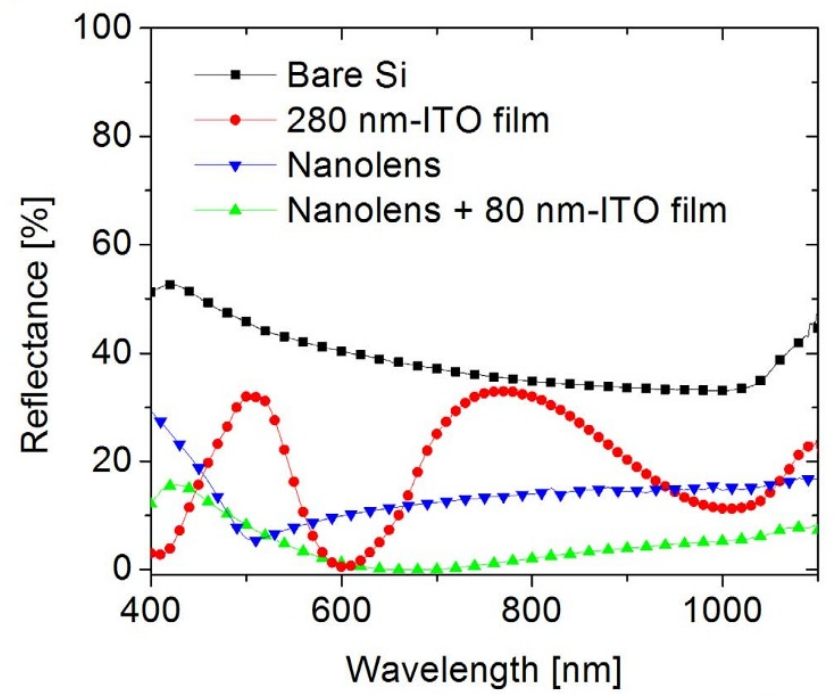

b

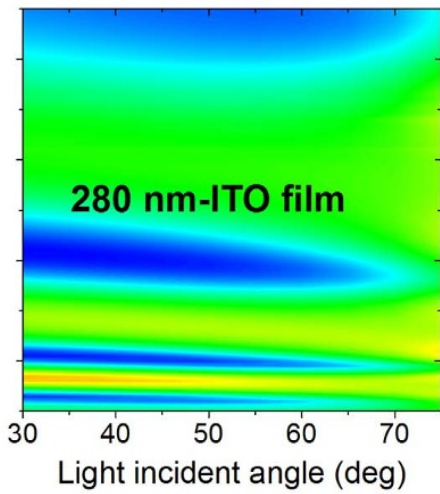

C

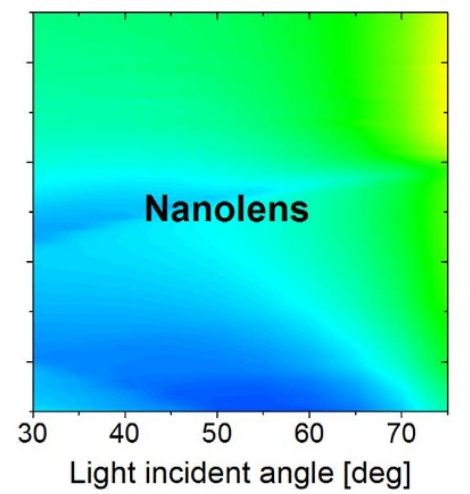

d

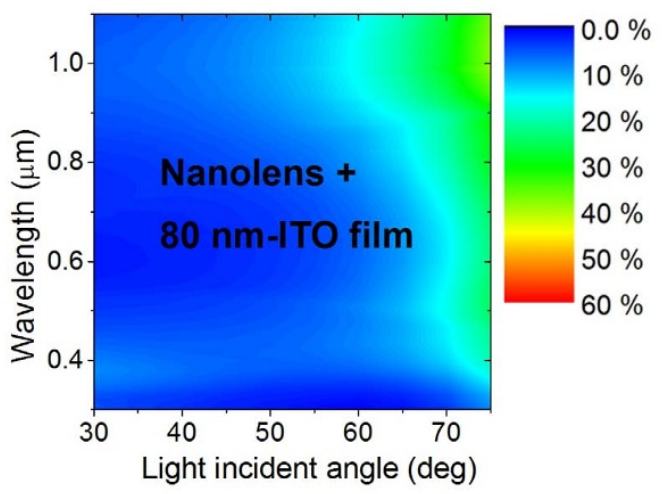

Figure $2 \mid$ Reflection profiles for the flat ITO film, nanolens-arrays, and nanolens-arrays with ITO film-coating on a Si substrate. (a), Nanolens-array with an additional ITO film coating significantly suppresses the reflection by $4.7 \%$ for $\lambda=400-1100 \mathrm{~nm}$. (b-d), Angle-dependent reflectance profiles as color maps.

calculations show the field pattern, consistent with expectations for the intermediate wavelengths. For light wavelengths $(\lambda)$ of $600 \mathrm{~nm}$ and $710 \mathrm{~nm}$, the Si absorber holds a moderate value of $\alpha_{\mathrm{si}}$. The first focusing peak appears close to the surface of the $\mathrm{Si}$ absorber. Neighboring nanolenses adjust the further light behavior, forming the second and third peak generations to the Si depth, (Fig. 3c,d). The second peak generation is distinctively found along the 'b-line', which is the center-point of neighboring nanolenses in the z-direction (Fig. 31). The third peak is formed along the 'a-line', which is a direction of a single nanolens in the z-direction (Fig. 3j). This light redirection phenomenon of incident light is uniquely observed for the ITO-nanolens structure, different from the multiple peak appearances of the tailored $\mathrm{Si}$ structure ${ }^{21}$ or the light propagation by scattering ${ }^{38}$.

Table 1 | Focal length profiles for various wavelengths. An ITOnanolens effectively modulates the incident light to cause longwavelengths to be shortened and short-wavelengths to be extended

\begin{tabular}{lcccc} 
Wavelength $(\mathrm{nm})$ & $\mathrm{n}_{\mathrm{ITO}}$ & $\mathrm{F}_{\text {air }}(\mathrm{nm})$ & $\mathrm{n}_{\mathrm{Si}}$ & $\mathrm{F}_{\text {lens }}(\mathrm{nm})$ \\
\hline 400 & 2.12 & 161.6 & 5.57 & 900.1 \\
500 & 1.88 & 205.6 & 4.29 & 882.3 \\
600 & 1.85 & 212.9 & 3.9 & 830.5 \\
710 & 1.82 & 220.7 & 3.77 & 832.2 \\
1100 & 1.76 & 238.1 & 3.54 & 843.1 \\
\hline
\end{tabular}

At a short-wavelength of $500 \mathrm{~nm}$, the $E_{\text {light }}$ intensity distribution shows a dominant first peak, close to an ITO-Si interface (Fig. 3j). No second or third peak can be clearly found at $\lambda=500 \mathrm{~nm}$ due to the higher $\alpha_{\text {si }}$ value at short-wavelengths, which results in fast decay of the field intensity (Fig. 3b).

For long-wavelengths (e.g., $\lambda=1,100 \mathrm{~nm}$ ), no significant peak can be clearly seen. The low refractive index of $\mathrm{Si}$ is the main physical origin that results in the weak lens effect. The incident photons are almost uniformly distributed in the Si absorber through the flat ITOfilm without redirection of the incident light ${ }^{16}$. The span of the $E$ is directly proportional to the wavelengths of the incident plane waves (Fig. 3f-i). The flat ITO-film surface does not have an effect of focusing the incident light and thus, the light absorption of $\mathrm{Si}$ is a function of the distance from the surface, at a fixed wavelength. For a long-wavelength, the period of the peak $E_{\text {light }}$ is extended compared to that of a short-wavelength. This is attributed to the increased span of the $E_{\text {light }}$ distribution according to the increases of wavelength. The long-wavelength photons induce relatively little contribution due to the low $\alpha_{\mathrm{si}}$ value for plane wave propagation. Meanwhile, the nanolens seems to be an appropriate solution to improve the utilization of long-wavelength photons for Si solar cells.

The generation rate of photo-carriers is proportional to the field intensity in a Si absorber. Thus, a spatial distribution of the photocarriers can be readily inferred from the $E_{\text {light }}$ intensity distributions. Practically, some of the photo-generated carriers are recombined, limiting the energy conversion efficiency of the solar cell. The SCR 

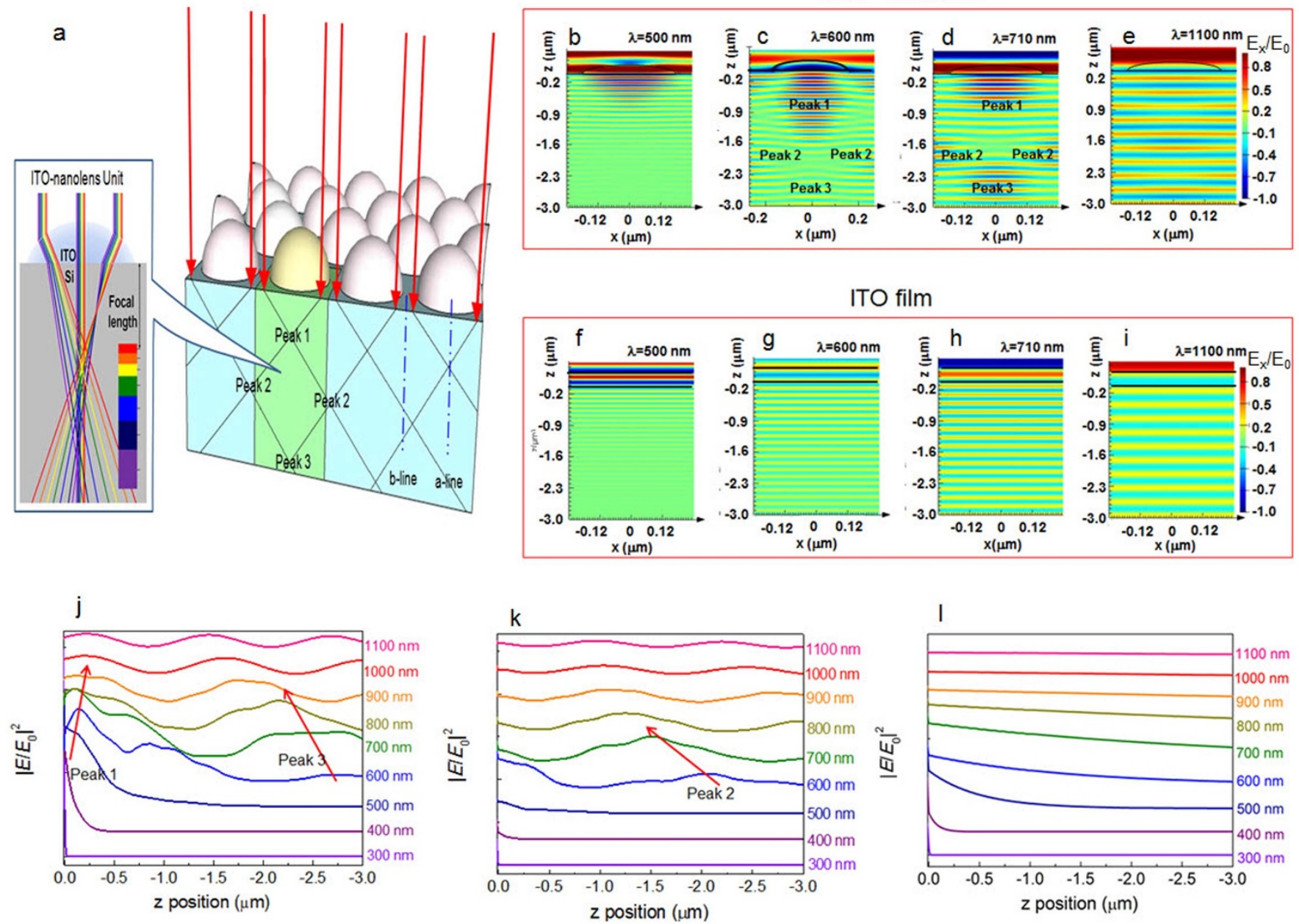

Figure 3 Light distribution according to wavelengths. (a), An ITO-nanolens effectively modulates the visible light into the Si absorber. (b-f), FDTD simulation shows strong electric distribution with ITO-nanolens. ( $\mathrm{f}-\mathrm{i})$, Incident light moves as plane waves through a flat ITO film. Strong electric peaks were observed along the (j) 'a-line' and (k) 'b-line'. (l) The flat ITO film shows only shallow electric fields for short wavelengths.

holds a strong built-in potential $(0.786 \mathrm{~V}$, Supplementary section 'Built-in potential'), which is a driving force to sweep the photogenerated minority carriers (electrons in $\mathrm{p}-\mathrm{Si}$ and holes in $\mathrm{n}-\mathrm{Si}$ ) in opposite directions ${ }^{11}$.

A strong built-in $E$ develops inside the SCR $\left(E_{S C R}\right)$ and the probability of photo-generated carrier collection is ideally unity in the SCR. Otherwise, a significant decay of the collection probability occurs in the neutral region ( $\mathrm{p}-\mathrm{Si}$ ) with an increasing distance from the SCR due to the exponential decrease of photo-generated electrons. In addition, the carriers should be diffusively collected without assistance of the $E_{S C R}$. Meanwhile, a heavily doped emitter layer (n$\mathrm{Si}$ ) is an easy spot for serious recombination of photo-generated carriers $^{11,29}$. This suggests an important clue. An enhanced performance solar cell may be achieved using a spatial overlapping of the high carrier generation region $\left(E_{\text {light }}\right)$ and the space of the high carrier collection probability of SCR $\left(E_{S C R}\right)$.

Solar cell performances. According to the doping profile, we have measured the emitter thickness and found it to be $394 \mathrm{~nm}$ (Fig. 4a, see Methods). Considering the heavy dopant concentrations $\left(10^{18} /\right.$ $\mathrm{cm}^{3}$ or higher), it is desirable to form a long $\mathrm{F}_{\text {lens }}$ beyond the emitter thickness to minimize the serious recombination loss at the surface. The SCR width of our cells was $572 \mathrm{~nm}$ (Supplementary section 'SCR analysis'). Therefore, an effective $E$ distribution should be positioned at a depth of 394-966 nm, where the SCR is located from the surface. The nanolens feature was designed with this consideration and fully satisfies the focal lengths in order to be positioned in the SCR for broad-band wavelengths (Table 1).

The geometric layout of the ITO-nanolens solar cell is presented as a schematic diagram (Fig. 4b). A relatively large-size nanolensembedded solar cell was prepared (Fig. 4c) and we characterized light-performance, under one-sun illumination (see Methods). The nanolens device provided substantially improved open circuit voltage $(590 \mathrm{mV})$, short circuit current $\left(35.82 \mathrm{~mA} / \mathrm{cm}^{2}\right)$, and a conversion efficiency (16.0\%) from $520 \mathrm{mV}, 27.80 \mathrm{~mA} / \mathrm{cm}^{2}$ and $10.9 \%$ efficiency of a flat ITO-film solar cell (Fig. 4d).

In what follows we discuss the main optical benefits of the ITOnanolens solar cell performance. Overall, the ITO-nanolens device has higher internal quantum efficiency (IQE) values for the broad wavelengths than did the planar device (Fig. 4e); this situation was different from that of the direct Si-etched structure ${ }^{12,52}$. A clear comparison of the two devices according to wavelength can be seen by plotting the relative IQE values of the ITO-nanolens over those of the ITO film (Fig. 4f). The ITO nanolens collimates light into the SCR and also is effective to reduce reflection.

The ITO film has an intrinsic free carrier loss for short and long-wavelengths, without creating electron-hole pairs ${ }^{53,54}$, causing low IQE values for the flat ITO film device. Otherwise, the ITO-nanolens provides improved performance according to the manipulation of the incident light for short, long, and visible wavelength light by propagation, concentration, and tuning effect, respectively. 
a

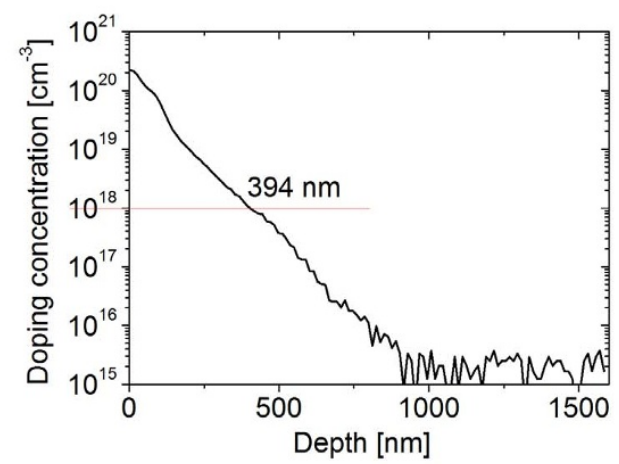

b

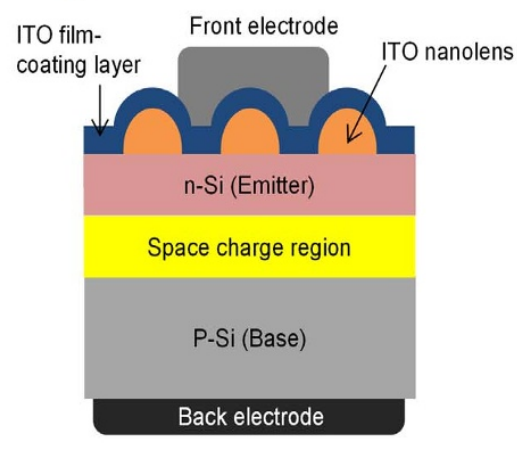

C

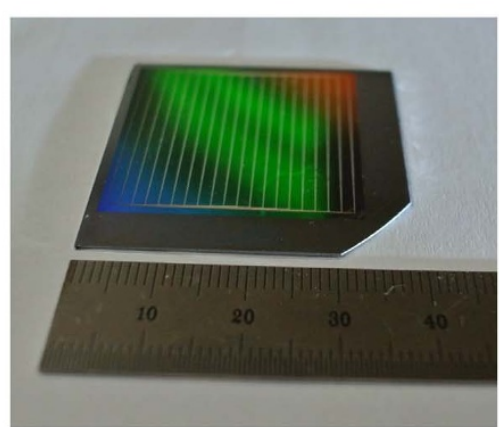

d

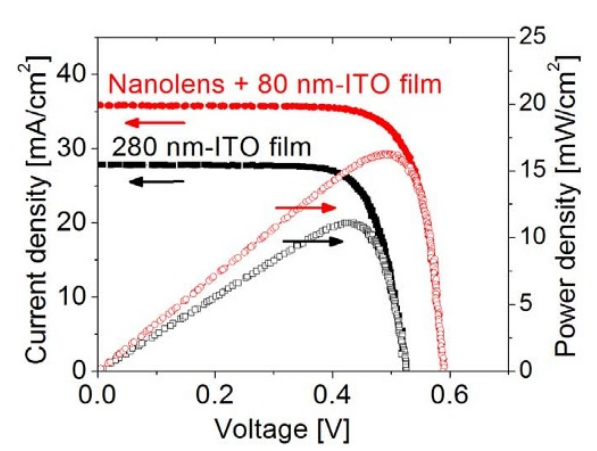

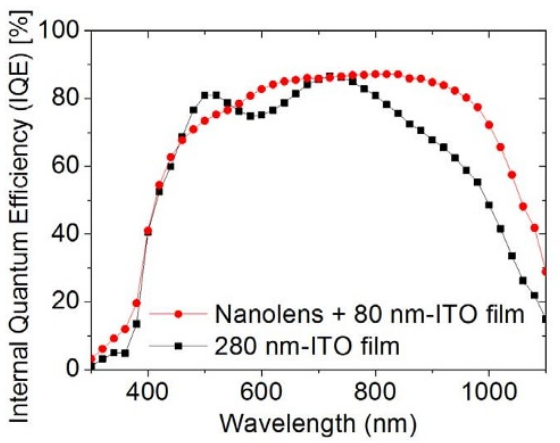

f

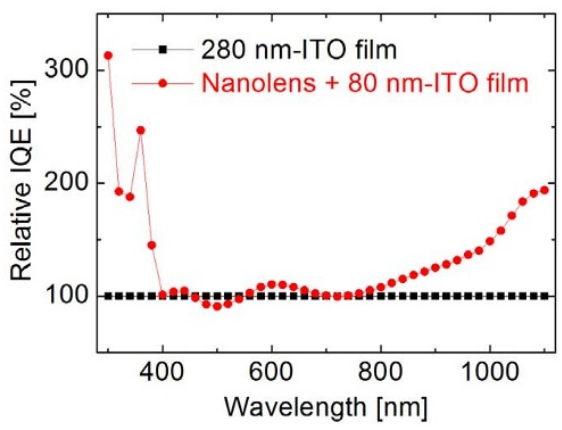

Figure 4 Solar cells: (a), Doping profile for emitter layer (see Methods). (b), Schematic of ITO-nanolens-embedded Si solar cell. (c), Photo of a fabricated solar cell. (d), Solar cell performances under one-sun illumination. (e-f), Comparison in quantum efficiencies for ITO-nanolens device and flat ITO device.

For short wavelengths, serious ITO loss can be relieved by passing the incident light through the nanolens to extend the short wavelength photon propagation into a deeper area of the Si. This significantly relieves the fast decay of the short-wavelength photons at the interface between the air and the ITO medium. Substantially enhanced IQE values were achieved at short-wavelengths. At $\lambda=$ $300 \mathrm{~nm}$, the IQE value of the ITO-nanolens was improved by $313 \%$ compared to that of the planar device. This is a distinctive advantage of the ITO-nanolens in that it can reduce the inherent ITO reflection loss for short-wavelengths ${ }^{21}$. The nanolens is effective in modulating the light propagation at short wavelengths, driving more photons to head towards the Si light-absorber.

For the visible range, the ITO-nanolens device showed generally improved IQE values. A 10\% enhanced IQE value was achieved for the ITO-nanolens device compared to that of the planar device at $\lambda=$ $600 \mathrm{~nm}$, which is the most important incident light for a Si lightabsorber ${ }^{11}$. The nanolens effectively modulates the visible wavelengths. Relatively long-wavelengths of visible light (red region, $\lambda=600-700 \mathrm{~nm})$ have shorter $F_{\text {lens }}$ values $(830.5-832.2 \mathrm{~nm})$ than that $(882.3 \mathrm{~nm})$ of blue light $(\lambda=500 \mathrm{~nm})$. The nanolens is efficient in tuning the visible-wavelength photons so that they are positioned in the SCR.

For long-wavelengths, the ITO-nanolens device shows prominently improved IQE performance, demonstrating an active scheme for low photon-energy utilization ${ }^{49}$. Light trapping of $800 \mathrm{~nm}-$ $1100 \mathrm{~nm}$ wavelengths is crucial for $\mathrm{Si}^{2}$. At $\lambda=1100 \mathrm{~nm}$, the IQE value of the ITO-nanolens was enhanced by $193.8 \%$. Incident light moves as a plane wave into the $\mathrm{Si}$ absorber through the ITO medium. For long-wavelengths, the $\mathrm{Si}$ absorber has insufficient absorption of long wave photons. Thus, a planar ITO film device identically bears a critical problem against a long collection length for minority carriers (electrons) in p-type Si. This typically causes an extremely low QE performance for infrared regimes ${ }^{55}$. Meanwhile, the ITO-nanolens efficiently drives long-wavelength photons to be focused near the SCR. This light-adjustable effect spontaneously resolves the low light absorption limit of $\mathrm{Si}$ at long- wavelengths and simultaneously establishes a short collection length. Due to an existing strong $E$ in the SCR, the photo-generated carriers can be effectively collected, resulting in improved $\mathrm{QE}$ values. The nanolens has a distinctive benefit in being able to concentrate the long-wavelengths into Si.

In summary, we propose a promising approach to nanostructure PVs by using a transparent ITO-nanolens without a direct etching of the semiconductor material and demonstrated a record high lightconversion efficiency of $16.0 \%$ among the periodic Si solar cells. A periodic structure of an ITO-nanolens was formed using a commercially viable printing method for large-scale devices. The ITO-nanolens provides optical and electrical benefits for nanostructured PVs. Due to the electrical conducting property, the ITO-nanolens supports the photo-generated carrier transport. For the optical aspects, the ITO-nanolens effectively adjusts the focal lengths for various light wavelengths, resulting in the overlapping of $E_{\text {light }}$ and $E_{S C R}$ to give a higher carrier collection efficiency. In previous work ${ }^{12}$, a microscale ITO lens was used. However, the efficiency improvement was not significant due to the off-positioned focal length location from SCR. Additionally, the ITO-nanolens can broaden near-zero reflection and provide high tolerance to the incident light angles. Ultimately, these benefits may enhance solar power-generation and utilization time. This optically transparent and electrically conductive nanolens architecture would be a promising scheme for the high-efficient nanostructure PVs.

\section{Methods}

The p-n junction formation. An emitter layer ( $\mathrm{n}-\mathrm{Si}$ ) was formed on a Czochralski (CZ) grown 4 -inch p-type (100) Si wafer, having a resistivity of $1-10 \Omega \mathrm{cm}$. 
Phosphoryl chloride $\left(\mathrm{POCl}_{3}\right)$ was used as a doping agent and was flowed into a doping furnace at $800^{\circ} \mathrm{C}$ for $40 \mathrm{~min}$. The phosphosilicate glass (PSG) formed during the doping step was removed using buffered hydrofluoric acid (10 wt\% HF).

ITO-coating and design. Nanolens-arrays were deployed on a Si substrate. An additional ITO-coating layer ( $2^{\text {nd }}$ ITO) was deposited to induce an electrical connection through all the nanolens entities. This is also an important detail that is used to control the high reflection of the bare Si region. A nanolens sitting on a Si region is optically affected by the ITO nanolens; however, a region not covered by an ITO nanolens displays the optical behavior of bare Si. Due to the intermediate refractive index of ITO film for air $(n=1)$ and $\mathrm{Si}(\mathrm{n}=3.54 \sim 5.57)$ systems, the insertion of an ITO film $(n=1.76 \sim 2.12)$ relieves the sudden changes of refractive indexes and therefore effectively diminishes the reflection. The optimum thickness of the $2^{\text {nd }}$ ITO film was determined to be $\sim 80 \mathrm{~nm}$, after considering a quarter wavelength anti-reflection scheme $(d=\lambda / 4 n)$, in which $n$ is the refractive index of ITO $(1.85$ at $\lambda=600 \mathrm{~nm})$. ITO depositions were performed in a dc-sputtering system using a 4-inch target composed of $\operatorname{In}_{2} \mathrm{O}_{2}$ containing $10 \mathrm{wt} \% \mathrm{SnO}_{2}$ at a working pressure of 5 mTorr under $\mathrm{Ar} / \mathrm{O}_{2}$ (50/1) ambient condition.

Calculation of focal lengths. The radius (R) of the ITO nanolens curvature can be achieved as in the following equation:

$$
\mathrm{R}=\frac{\mathrm{h}^{2}+\mathrm{r}^{2}}{2 \mathrm{~h}}
$$

where $h$ is the height of the ITO nanolens $(200 \mathrm{~nm})$ and $r$ is the radius $(180 \mathrm{~nm})$. Considering these values, we have achieved an $\mathrm{R}$ value of $181 \mathrm{~nm}$. In air conditions, the focal length $\left(\mathrm{F}_{\mathrm{air}}\right)$ of the ITO nanolens can be found using the following equation:

$$
\mathrm{F}_{\mathrm{air}}=\frac{\mathrm{R}}{\mathrm{n}_{\mathrm{ITO}}-\mathrm{n}_{\mathrm{air}}}
$$

When we consider a Si medium, the focal length is multiplied by the refractive index of $\mathrm{Si}\left(\mathrm{n}_{\mathrm{si}}\right)$. And thus, the effective focal length of the ITO nanolens $\left(\mathrm{F}_{\text {lens }}\right)$ on Si can be extended according to the following sequence ${ }^{46}$ :

$$
\mathrm{F}_{\text {lens }}=\mathrm{F}_{\text {air }} \times \mathrm{n}_{\mathrm{Si}}
$$

Emitter layer determination. The emitter depth was measured by secondary ion mass spectroscopy (SIMS, Cameca, magnetic sector ims7f). Regarding the $\mathrm{p}-\mathrm{Si}$ doping level $\left(\sim 10^{16} / \mathrm{cm}^{3}\right)$, a two order higher level $\left(10^{18} / \mathrm{cm}^{3}\right)$ was chosen for the ntype doping region in order to give a $394 \mathrm{~nm}$-thickness to the emitter (Fig. 4a).

Device fabrication and light responses. A flat ITO device and a nanolens device were tailored to a size of $3.2 \times 3.2 \mathrm{~cm}^{2}$. Solar cell performances were obtained under onesun illumination using a simulator (McScience, K3000). The profiles of the carrier collection, according to wavelength variations, were obtained using a quantum efficiency measurement system (McScience, K3100).

1. Kim, J. et al. Three-Dimensional a-Si:H Solar Cells on Glass Nanocone Arrays Patterned by Self-Assembled Sn Nanospheres. ACS Nano 6, 265-271 (2011).

2. Wang, K. X. et al. Absorption Enhancement in Ultrathin Crystalline Silicon Solar Cells with Antireflection and Light-Trapping Nanocone Gratings. Nano Lett. 12, 1616-1619 (2012).

3. Ferry, V. E., Polman, A. \& Atwater, H. A. Modeling Light Trapping in Nanostructured Solar Cells. ACS Nano 5, 10055-10064 (2011).

4. Kwon, J. Y. et al. High Efficiency Thin Upgraded Metallurgical-Grade Silicon Solar Cells on Flexible Substrates. Nano Lett. 12, 5143-5147 (2012).

5. Adachi, M. M., Anantram, M. P. \& Karim, K. S. Core-shell silicon nanowire solar cells. Sci. Rep. 3 (2013).

6. Mavrokefalos, A. et al. Efficient Light Trapping in Inverted Nanopyramid Thin Crystalline Silicon Membranes for Solar Cell Applications. Nano Lett. 12, 2792-2796 (2012).

7. Jung, Y. et al. Record High Efficiency Single-Walled Carbon Nanotube/Silicon pn Junction Solar Cells. Nano Lett. 13, 95-99 (2012).

8. Cao, L. et al. Semiconductor Nanowire Optical Antenna Solar Absorbers. Nano Lett. 10, 439-445 (2010).

9. Krogstrup, P. et al. Single-nanowire solar cells beyond the Shockley-Queisser limit. Nat. Photonics 7, 306-310 (2013).

10. Hadiseh, A., Ashwin, C. A. \& Jennifer, A. D. Optimized light absorption in Si wire array solar cells. J. Opt. 14, 024006 (2012).

11. Kim, H. et al. Effect of the short collection length in silicon microscale wire solar cells. Appl. Phys. Lett. 102, 193904 (2013).

12. Kim, J. et al. Effective light management of three-dimensionally patterned transparent conductive oxide layers. Appl. Phys. Lett. 101, 143904 (2012).

13. Kim, J. et al. Surface-concentrated light and efficient carrier collection in microhole-patterned Si solar cells. Opt. Express 21, A607-A615 (2013).

14. Dutto, F. et al. Enhancement of Second Harmonic Signal in Nanofabricated Cones. Nano Lett. 13, 6048-6054 (2013).
15. Tan, H., Santbergen, R., Smets, A. H. M. \& Zeman, M. Plasmonic Light Trapping in Thin-film Silicon Solar Cells with Improved Self-Assembled Silver Nanoparticles. Nano Lett. 12, 4070-4076 (2012).

16. Vasudev, A. P., Schuller, J. A. \& Brongersma, M. L. Nanophotonic light trapping with patterned transparent conductive oxides. Opt. Express 20, A385-A394 (2012).

17. Li, Y. et al. Novel Silicon Nanohemisphere-Array Solar Cells with Enhanced Performance. Small 7, 3138-3143 (2011).

18. Zhu, J. et al. Nanodome Solar Cells with Efficient Light Management and SelfCleaning. Nano Lett. 10, 1979-1984 (2009).

19. Gjessing, J., Sudbø, A. S. \& Marstein, E. S. Comparison of periodic light-trapping structures in thin crystalline silicon solar cells. J. Appl. Phys. 110, 033104 (2011).

20. Herman, A. et al. Influence of the pattern shape on the efficiency of front-side periodically patterned ultrathin crystalline silicon solar cells. J. Appl. Phys. 112, 133107-133107-133108 (2012).

21. Biswas, R. \& Xu, C. Nano-crystalline silicon solar cell architecture with absorption at the classical 4n2 limit. Opt. Express 19, A664-A672 (2011)

22. Service, R. F. Can the Upstarts Top Silicon? Science 319, 718-720 (2008).

23. Polman, A. \& Atwater, H. A. Photonic design principles for ultrahigh-efficiency photovoltaics. Nat. Mater. 11, 174-177 (2012).

24. Priolo, F., Gregorkiewicz, T., Galli, M. \& Krauss, T. F. Silicon nanostructures for photonics and photovoltaics. Nat. Nanotechnol. 9, 19-32 (2014).

25. Zongfu, Y., Raman, A. \& Fan, S. Fundamental Limit of Light Trapping in Grating Structures. Opt. Exp. 18, A366-380 (2010).

26. Battaglia, C. et al. Light Trapping in Solar Cells: Can Periodic Beat Random? ACS Nano 6, 2790-2797 (2012).

27. Jeong, S., McGehee, M. D. \& Cui, Y. All-back-contact ultra-thin silicon nanocone solar cells with $13.7 \%$ power conversion efficiency. Nat. Commun. 4, 2950 doi: 10.1038/ncomms3950 (2013).

28. Seo, K. et al. Si Microwire Solar Cells: Improved Efficiency with a Conformal SiO2 Layer. ACS Nano 7, 5539-5545 (2013).

29. Oh, J., Yuan, H.-C. \& Branz, H. M. An 18.2\%-efficient black-silicon solar cell achieved through control of carrier recombination in nanostructures. Nat. Nanotechnol. 7, 743-748 (2012).

30. Wallentin, J. et al. InP Nanowire Array Solar Cells Achieving 13.8\% Efficiency by Exceeding the Ray Optics Limit. Science 339, 1057-1060 (2013).

31. Qing Wan, Dattoli, E. N., Fung, W. Y., Wei Guo, Yanbin Chen, Xiaoqing Pan \& Wei Lu. High-Performance Transparent Conducting Oxide Nanowires. Nano Lett. 6, 2909-2915 (2006).

32. Jing Gao, Lebedev, O. L., Stuart Turner, Yong Feng Li, Yun Hao Lu, Yuan Ping Feng, Philippe Boullay, Wilfrid Prellier, Gustaaf van Tendeloo \& Tom Wu. Phase Selection Enabled Formation of Abrupt Axial Heterojunctions in Branched Oxide Nanowires. Nano Lett. 12, 275-280 (2012).

33. Dwyer, C. O., Szachowicz, M., Visimberga, G., Lavayen, V., Newcomb, S. B. \& Sotomayor Torres, C. M. Bottom-up growth of fully transparent contact layers of indium tin oxide nanowires for light-emitting devices. Nat. Nanotechnol. 4, 239-244 (2009).

34. Bozhi, T. et al. Coaxial silicon nanowires as solar cells and nanoelectronic power sources. Nature 449, 885-889 (2007).

35. Lee, E. et al. Comparative experimental and simulative investigations of radial $\mathrm{p}-\mathrm{n}$ junction Si microwire array solar cells. Sol. Energ. Mat. Sol. Cells 103, 93-97 (2012).

36. Service, R. F. Performance of Nanowire Solar Cells on the Rise. Science 339, 263 (2013).

37. Tatsuo, S. Advances in crystalline silicon solar cell technology for industrial mass production. NPG Asia Mater. 2, 96-102 (2010).

38. Ferry, V. E., Munday, J. N. \& Atwater, H. A. Design considerations for plasmonic photovoltaics. Adv. Mater. 22, 4794-4808 (2010).

39. Li, K., Stockman, M. I. \& Bergman, D. J. Self-Similar Chain of Metal Nanospheres as an Efficient Nanolens. Phys. Rev. Lett. 91, 227402 (2003).

40. Kawata, S., Ono, A. \& Verma, P. Subwavelength colour imaging with a metallic nanolens. Nat. Photonics 2, 438-442 (2008).

41. Cho, Y., Gwon, M., Park, H. H., Kim, J. \& Kim, D. W. Wafer-scale nanoconical frustum array crystalline silicon solar cells: promising candidates for ultrathin device applications. Nanoscale 6, 9568-9573 (2014).

42. Hong, P. S. \& Lee, H. H. Pattern uniformity control in room-temperature imprint lithography. Appl. Phys. Lett. 83, 2441-2443 (2003).

43. Miyadera, T. et al. Analytical model for the design principle of large-area solar cells. Sol. Energ. Mat. Sol. Cells 97, 127-131 (2012).

44. Meng, X. et al. Combined front and back diffraction gratings for broad band light trapping in thin film solar cell. Opt. Express 20, A560-A571 (2012).

45. Munday, J. N. \& Atwater, H. A. Large Integrated Absorption Enhancement in Plasmonic Solar Cells by Combining Metallic Gratings and Antireflection Coatings. Nano Lett. 11, 2195-2201 (2010).

46. Ma, C. \& Liu, Z. Focusing light into deep subwavelength using metamaterial immersion lenses. Opt. Express 18, 4838-4844 (2010).

47. Poon, P. C. H., Commander, L. G., Selviah, D. R. \& Robinson, M. G. Extension of the useful focal length range of microlenses by oil immersion. J. Opt. A-Pure Appl. Op. 1, 133-141 (1999).

48. Wu, Q., Feke, G. D., Grober, R. D. \& Ghislain, L. P. Realization of numerical aperture 2.0 using a gallium phosphide solid immersion lens. Appl. Phys. Lett. 75, 4064-4066 (1999). 
49. Tvingstedt, K., Dal Zilio, S., Inganäs, O. \& Tormen, M. Trapping light with micro lenses in thin film organic photovoltaic cells. Opt. Express 16, 21608-21615 (2008).

50. Koyama, K. et al. High collection efficiency in fluorescence microscopy with a solid immersion lens. Appl. Phys. Lett. 75, 1667-1669 (1999).

51. Lin, C. \& Povinelli, M. L. Optical absorption enhancement in silicon nanowire arrays with a large lattice constant for photovoltaic applications. Opt. Express 17, 19371-19381 (2009).

52. Chen, T.-G. et al. Characteristics of large-scale nanohole arrays for thin-silicon photovoltaics. Prog. Photovolt. Res. Appl. (2012).

53. Holman, Z. C. et al. Infrared light management in high-efficiency silicon heterojunction and rear-passivated solar cells. J. Appl. Phys. 113, 013107 (2013).

54. Holman, Z. C. et al. Current Losses at the Front of Silicon Heterojunction Solar Cells. IEEE J. Photovolt. 2, 7-15 (2012).

55. Subrahmanyam, A. \& Balasubramanian, N. Studies of the photovoltaic behaviour of indium tin oxide (ITO)/silicon junctions prepared by the reactive thermal evaporation technique. Semicond. Sci. Technol. 7, 324-327 (1992).

\section{Acknowledgments}

The authors acknowledge the financial support of the Korea Institute of Energy Technology Evaluation and Planning, in a grant funded by the Ministry of Knowledge and Economy (KETEP-20133030011000).

\section{Author contributions}

J.K. conceived this research. J.H.Y. designed the ITO-nanolens geometry and fabricated solar cell devices. J.Y. analyzed device performances. H.H.P. performed the nanoimprint method to fabricate the ITO-nanolens-arrays. E.L. performed FDTD simulation. J.Z. and J.S. performed the ellipsometric measurement. D.W.K. supervised E.L. W.A.A. supervised J.-H. N.M.L. supervised J.Z. J.K., D.W.K., W.A.A. and N.M.L. participated in discussion throughout the work and intensively cooperated to analyze results. M.M.D. Kumar analyzed QE performances. All the authors contributed to prepare this manuscript.

\section{Additional information}

Supplementary information accompanies this paper at http://www.nature.com/ scientificreports

Competing financial interests: The authors declare no competing financial interests.

How to cite this article: Yun, J.-H. et al. Incident light adjustable solar cell by periodic nanolens architecture. Sci. Rep. 4, 6879; DOI:10.1038/srep06879 (2014).

This work is licensed under a Creative Commons Attribution 4.0 International License. The images or other third party material in this article are included in the article's Creative Commons license, unless indicated otherwise in the credit line; if the material is not included under the Creative Commons license, users will need to obtain permission from the license holder in order to reproduce the material. To view a copy of this license, visit http://creativecommons.org/licenses/by/4.0/ 